\title{
INITIAL AND UNIVERSAL METRIC SPACES
}

\author{
W. HOLSZTYŃSKI ${ }^{1}$
}

\begin{abstract}
Local capacity is introduced (the usual notion of the capacity is global). It is proven (see Theorem 1) that some classes of metric spaces, naturally defined in terms of local capacity, contain a space which can be mapped onto any other member of its class without any stretching. Such a theorem would fail if local capacity is replaced by the usual notion of (global) capacity.

Using Theorem 1 and simple properties of Met $(X, Y)$ (see \$2) it follows immediately that for every class of compact metric spaces with uniformly bounded diameters and capacities there exists a compact space which contains an isometric image of any space from the class (in general this universal space cannot be found within the class).
\end{abstract}

Introduction. Today it is well known that the global notions of metric capacity are useful in the theory of approximation, functional analysis and some other branches of mathematics (geometry of numbers, topology, ....). Capacity of a metric space $X$ can be defined as a function $\varepsilon \rightarrow S_{\varepsilon}(X), \varepsilon>0$, where $S_{\varepsilon}(X)$ is the smallest cardinal number of a cover of $X$ with the subsets of diameter $\leqslant \varepsilon$. In this paper a local notion of metric capacity is introduced. There are some reasons to expect that such local notions should be very often even more convenient than global ones. The goal of this paper is purely metric. In $\$ 1$ we prove that every class of complete metric spaces with uniformly bounded diameters and local capacity contains a member which can be mapped onto any other one without increasing any distance (see Theorem 1 below). The following simple example shows that such a theorem would be false for global capacity. Let $X=\left\{x_{1}, x_{2}, x_{3}, x_{4}\right\}$ with metric $d\left(x_{1}, x_{i}\right)=2$ for $i=2,3,4$ and $d\left(x_{i}, x_{j}\right)=1$ for $2 \leqslant i<j \leqslant 4$, and $Y=\left\{y_{1}, y_{2}, y_{3}, y_{4}\right\}$ with metric $d\left(y_{1}, y_{2}\right)=d\left(y_{3}, y_{4}\right)=1$ and $d\left(y_{i}, y_{j}\right)=2$ for $i=1$ or 2 and $j=3$ or 4. Both $X$ and $Y$ can be covered by two sets of diameter $\leqslant 1$ and by four sets of diameter $\leqslant \frac{1}{2}$. But if a metric space $Z$ is a union of two subsets of diameter $\leqslant 1$ and if $Z$ can be mapped metrically (i.e., without increasing distances) onto $X$ and $Y$, then $Z$ cannot be covered by four subsets of diameter $\leqslant \frac{1}{2}$.

In $\S 2$ we apply Theorem 1 to prove a kind of dual theorem on universal metric spaces (we use a contravariant functor Met from the category of metric spaces into itself).

1. A mapping $f: X \rightarrow Y$ ( $X, Y$ being metric spaces) such that

Received by the editors September 30, 1974.

AMS (MOS) subject classifications (1970). Primary 54E40; Secondary 46E99.

1 While writing this paper the author was partially supported by a NSF grant. 


$$
d(f(x), f(y)) \leqslant d(x, y) \text { for } x, y \in X
$$

is called a metric mapping.

Let $M$ be a class of metric spaces. Then a relation $\geqslant$, defined by $X \geqslant Y$ iff there exists a metric map onto $f: X \rightarrow Y$, is a partial quasi-ordering of $M$. If $M$ consists only of compact spaces then $\geqslant$ is a partial ordering up to isometry (i.e. from $X \geqslant Y$ and $Y \geqslant X$ it follows that $X$ and $Y$ are isometric), see [1].

Definition 1. Space $X$ is an initial space of $M$ iff $X \geqslant Y$ for every $Y \in M$.

We shall show that for some naturally distinguished classes there exist initial spaces, but we will begin with some simple propositions.

Proposition 1. If $X \geqslant Y$ then $\operatorname{card} X \geqslant \operatorname{card} Y$ and $\operatorname{diam} X \geqslant \operatorname{diam} Y$.

Proposition 2. If a class $M$ has an initial space then there exists a cardinal number $m$ such that $\operatorname{card} X \leqslant m$ for every $X \in M$.

Proposition 3. If $M$ is the class consisting of all spaces $X$ with card $X \leqslant m$ and $\operatorname{diam} X \leqslant a$ then the space $D(m, a)$ with card $D(m, a)=m$ and with metric given by

$$
d(x, y)=\left\{\begin{array}{l}
0 \text { for } x=y, \\
a \text { for } x \neq y,
\end{array} \quad x, y \in D(m, a),\right.
$$

is an initial space of $M$.

PRoposition 4. If $M$ contains only bounded spaces with arbitrarily great diameters then $M$ does not possess any initial space.

Now let $N(X, k)$ be the least cardinal number such that for every subset $A$ of $X$ with $\operatorname{diam} A \leqslant 2^{k}$ there exists a covering $C$ of $A$, consisting of subsets with diameters $\leqslant 2^{k-1}$, such that card $C \leqslant N(X, k)$. Now let $M\left(m_{k}: k\right.$ $=0, \pm 1, \ldots)$ be the class of all complete metric spaces $X$ such that $N(X, k)$ $\leqslant m_{k}$ for every integer $k$.

REMARK. If $N(X, k)=1$ for all integers $k \geqslant n+1$ (n-an integer) then $\operatorname{diam} X \leqslant 2^{n}$.

The purpose of this note is the following theorem (compare [2]).

THEOREM 1. Let $\left(m_{k}: k=0, \pm 1, \ldots\right)$ be a sequence of cardinal numbers such that $m_{k}=1$ for $k \geqslant n+1$, where $n$ is an integer. Then class $M=M\left(m_{k}: k\right.$ $=0, \pm 1, \ldots)$ has an initial space. For example, cartesian product

$$
D=\prod_{-\infty}^{\infty} D\left(m_{k}, 2^{k}\right)
$$

(see Proposition 3), with metric

$$
d(x, y)=\max \left(d\left(x_{k}, y_{k}\right): k=0, \pm 1, \ldots\right)
$$

(for every $x=\left(x_{k}\right)_{k=0, \pm 1, \ldots}$ and $\left.y=\left(y_{k}\right)_{k=0, \pm 1, \ldots} \in D\right)$ is an initial space of $M$.

Proof. We have to show that $D \geqslant X$ for every $X \in M$. Let $C_{k}(A)$ be a covering of $A$ consisting of closed subsets of diameters $\leqslant 2^{k-1}$ and with 
card $C_{k}(A) \leqslant m_{k}$, for every $A \in M$ with $\operatorname{diam} A \leqslant 2^{k}$. We can assume $C_{k}(A)=\{A\}$ for $k \geqslant n+1$.

Now let $X \in M$ be a fixed space. For $k \geqslant n+1$ we let

$$
F_{k, x^{k}}: D\left(m_{k}, 2^{k}\right) \rightarrow\{X\}=C_{k}(X)
$$

be the unique function that exists and we define

$$
F_{k, x^{k}}: D\left(m_{k}, 2^{k}\right) \rightarrow C_{k}\left(F_{k+1, x^{k+1}}\left(x_{k+1}\right)\right),
$$

as an arbitrary mapping onto, for every integer $k$ and $x^{k}=\left(x_{k}, x_{k+1}\right.$, $\left.x_{k+2}, \ldots\right) \in \prod_{i \geqslant k} D\left(m_{i}, 2^{i}\right)$ and $x^{k+1}=\left(x_{k+1}, x_{k+2}, \ldots\right)$.

Thus we have recursively defined a sequence of functions $\cdots, F_{-1, x^{-1}}$, $F_{0, x^{0}}, F_{1, x^{1}}, \ldots$ such that $F_{k, x^{k}}\left(x_{k}\right) \supseteq F_{k-1, x^{k-1}}\left(x_{k-1}\right)$ for every $k$ and $x \in D$. Let $f(x)$ be the unique point of the intersection $\cap_{k=-\infty}^{\infty} F_{k, x^{k}}\left(x_{k}\right)$. We shall show that the function $f: D \rightarrow X$ defined in such a way is a metric mapping.

Indeed, let $x, y \in D$ and let $k$ be the maximal integer such that $x_{k} \neq y_{k}$. Then $k \leqslant n$ and $d(x, y)=2^{k}$. On the other hand $F_{i, x^{i}}=F_{i, y^{i}}$ for $i>k$. Hence

$$
f(x), f(y) \in F_{k+1, x^{k+1}}\left(x_{k+1}\right)=F_{k+1, y^{k+1}}\left(y_{k+1}\right)
$$

and

$$
d(f(x), f(y)) \leqslant \operatorname{diam} F_{k+1, x^{k+1}}\left(x_{k+1}\right) \leqslant 2^{k}=d(x, y) .
$$

Thus $f$ is a metric mapping. Now we shall show that $f$ is onto $X$.

Indeed, let $p \in X$. We let $x_{k}$ be the unique element of $D\left(m_{k}, 2^{k}\right)$ for $k \geqslant n+1$ and let it be an arbitrary element of $D\left(m_{k}, 2^{k}\right)$ such that $p$ $\in F_{k, x^{k}}\left(x_{k}\right)$ for every $k \leqslant n$. Thus we have a recursive definition of a point $x=\left(x_{k}\right)_{k=0, \pm 1, \ldots} \in D$ such that $f(x)=p$.

It remains to prove $D \in M$. Let $A \subseteq D$ and $\operatorname{diam} A \leqslant 2^{k}$. Then for every $x=\left(x_{i}\right)_{i=0,+1, \ldots, y}=\left(y_{i}\right)_{i=0, \pm 1, \ldots} \in A$ we have $x_{i}=y_{i}$ for $i \geqslant k+1$, i.e. $x^{k+1}=y^{k+\Gamma}$. Hence there exists $a^{k+1}=\left(a_{k+1}, a_{k+2}, \ldots\right)$ such that $x^{k+1}$ $=a^{k+1}$ for every $x \in A$. We can put

$$
A_{q}=\left\{x \in A: X^{k+1}=a^{k+1} \text { and } x_{k}=q\right\} \text { for } q \in D\left(m_{k}, 2^{k}\right) .
$$

Evidently $\operatorname{diam} A_{q} \leqslant 2^{k-1}$ and $C=\left\{A_{q}: q \in D\left(m_{k}, 2^{k}\right)\right\}$ is a covering of $A$ such that $C=m_{k}$.

REMARK 1. If $X$ and $Y$ are compact metric spaces such that $X \geqslant Y$ and $Y \geqslant X$ then it is well known that $X$ and $Y$ are isometric. Thus if $m_{k}<\infty$ for every integer $k$ then the initial space for $M=M\left(m_{k}: k=0, \pm 1, \ldots\right)$ is unique up to isometry.

REMARK 2. Theorem 1 implies the well-known theorems: (i) every complete and totally bounded metric space is compact, (ii) every metric compact space is a continuous image of the Cantor Discontinuum $C=\prod_{k=0}^{\infty} D\left(2,2^{-k}\right)$, (iii) every complete separable metric space is a continuous image of the space of irrational numbers.

Indeed, if $X$ is complete and totally bounded then there is a sequence of 
integers $\left(m_{k}: k=0, \pm 1, \ldots\right)$ such that $N(X, k) \leqslant m_{k}$ for $k=0, \pm 1, \ldots$ Then $X$ is a continuous image of $D=\prod D\left(m_{k}, 2^{k}\right)$. Thus $X$ is compact. Furthermore, we can assume that each $m_{k}$ is a power of 2, i.e., that $D\left(m_{k}, 2^{k}\right)$ is homeomorphic to the cartesian product of finitely many 2-point spaces. Thus $D$ is homeomorphic to the Cantor Discontinuum.

Now let $X$ be an arbitrary complete metric space. We can assume in addition that $\operatorname{diam} X \leqslant 1$. Then $X$ is a metric image of $D=D\left(m_{k} ; 2^{k}\right)$ where $m_{k}=0$ for positive $k$ and $m_{k}$ is countable infinite for $k \leqslant 0$. But $D$ is homeomorphic to the space of irrational numbers.

2. In 1961 A. Pełczyński conjectured and the author proved that

For every class of compact spaces with uniformly bounded diameters and (global) capacities there exists a universal (in the sense of isometric embeddings) compact space. ${ }^{2}$

One of two proofs the author gave (but never published) required a version of Theorem 1 of this paper, with local capacity replaced by the global one (see [3]). Since versions with global and local capacity of Theorem (*) are equivalent, we will use the local capacity. The following notion of Met $(X, Y)$ and its basic properties (including a metric version of Banach-Stone's theorem) were obtained by the author in 1961/62 (see [2]).

Given metric spaces $X, Y$ let Met $(X, Y)$ be the set of all metric maps of $X$ into $Y$, with the uniform metric

$$
d(f, g)=\sup (d(f(x), g(x)): x \in X) .
$$

In general Met $(X, Y)$ is a generalized metric space (sometimes $d(f, g)=\infty$ ), but if $X$ or $Y$ is bounded then $d(f, g)<\infty$ for every $f, g \in \operatorname{Met}(X, Y)$. If both $X, Y$ are compact then Met $(X, Y)$ is compact by Arzela's theorem).

Property 1. Let $\phi: X_{1} \rightarrow X_{2}$ be a metric map. Then function

$$
\Phi: \operatorname{Met}\left(X_{2}, Y\right) \rightarrow \operatorname{Met}\left(X_{1}, Y\right)
$$

given by $\Phi(f)=f \circ \phi$ is metric. Moreover, if $\phi$ is onto (or onto a dense subset of $X_{2}$ ) then $\Phi$ is an isometric embedding.

ProperTY 2. Let $X$ be a metric space and $I \supseteq[0$; diam $X] \backslash\{\infty\}$ be an interval of reals. Then $X$ can be isometrically embedded in Met $(X, I)$. An embedding $i: X \rightarrow \operatorname{Met}(X, I)$ is given by $(i(x))(y)=d(x, y)$ for $x, y \in X$ (cf. [5], [4]).

Combining Properties 1,2 and Theorem 1 from $\S 1$, we obtain the following generalized version of $(*)$.

THEOREM 2. Every space of $M$ can be isometrically embedded in $U$ $=\operatorname{Met}\left(D,\left[0 ; 2^{n}\right]\right)$, where $M, n, D$ are as in Theorem 1. If, in addition, all cardinals $m_{k}, k=0, \pm 1, \ldots$, are finite then $U$ is compact.

\section{REFERENCES}

1. H. Freudenthal and W. Hurewicz, Dehnungen, Verkürzungen, Isometrien, Fund. Math. 26 (1936), 120-122.

${ }^{2}$ In general, but in very special cases, such a universal space cannot belong to the class. 
2. W. Holsztyński, $O$ przestrzeniach metrycznych. I, (On metric spaces. I), Work presented on the J. Marcinkiewicz VI competition, 1961-1962. (Polish)

3. - Complete metric spaces as the quasi-open images of the Baire's spaces, Bull. Acad. Polon. Sci. Sér. Sci. Math. Astronom. Phys. 12 (1964), 743-745. MR 30 \# 2463.

4. —, On metric spaces aimed at their subspaces, Prace Mat. 10 (1966), 95-100. MR 33 \#4895.

5. K. Kuratowski, Quelques problèmes concernant les espaces métriques non-séparables, Fund. Math. 25 (1935), 534-545.

Department of Mathematics, University of Western Ontario, London, Ontario, CanaDA 